\title{
Gunshot tibia fractures treated with intramedullary nailing: A single centre retrospective review
}

\author{
TL Hilton MBChB(UCT), DA(SA), DipPEC(SA), FCOrth(SA), MMed(UCT) \\ Orthopaedic Consultant, Groote Schuur Hospital, University of Cape Town \\ N Kruger MBChB(UCT), MSc Diag Imag(Oxon), MSc(res) Orth Surg(Oxon) \\ Orthopaedic Registrar, Groote Schuur Hospital, University of Cape Town \\ KR Wiese MBChB(UCT) \\ Orthopaedic Medical Officer, Worchester Provincial Hospital, Cape Town \\ CW Martin BA(North Western), MD(Texas), MSc(Oxon)
}

Orthopaedic Surgery Resident, University of Texas Health Science Center, San Antonio, United States of America

S Maqungo MBChB(Natal), FCOrth(SA), MMed(UCT)

Consultant Orthopaedic Surgeon, Orthopaedic Trauma Service, Groote Schuur Hospital, University of Cape Town

\author{
Corresponding author: \\ Dr Thomas L Hilton \\ 11 Clive Street \\ Vredehoek \\ 8000 Cape Town \\ South Africa
}

Email: tlhilton@hotmail.com

Cell: 0027 (82) 7967608

\begin{abstract}
Background: Open tibia fractures are notoriously difficult to treat, with a high rate of union problems and infection. Gunshot wound-associated fractures of the tibia compound these issues further by causing extensive bone comminution and soft tissue damage. No universally accepted management protocol exists, but intramedullary (IM) nailing of these injuries is an attractive treatment strategy. It provides stable internal fixation and limits further insult to the soft tissue envelope. It also allows complete access for wound management and early range of movement of the adjacent joints. This study aims to review the results of patients treated with IM nailing for gunshot wound (GSW) tibia fractures to assess whether this is a viable treatment option for this injury.

Methods: A retrospective folder review was performed of all adult patients who sustained a GSW tibia fracture treated with intramedullary nailing between January 2009 and December 2014. Parameters evaluated included time to theatre, time to wound closure, radiographic extent of fracture comminution, anatomical alignment, time to union and incidence of chronic osteomyelitis.

Results: Twenty-two patients were eligible for inclusion; however, nine were lost to follow-up. The remaining 13 patients achieved union over an average of 26 weeks. Three cases developed osteomyelitis, all of which had radiographic zones of comminution exceeding $120 \mathrm{~mm}$. No cases of malunion were reported and no other significant trends noted.

Conclusion: Treatment of tibial gunshot fractures must be individualised according to both the soft tissue injury and radiographic zone of comminution in order to achieve a favourable outcome. Intramedullary nailing is an effective treatment strategy for low Gustilo-Anderson grade injuries, with minimal complications.
\end{abstract}

Key words: gunshot wound, intramedullary nailing, tibia fracture 


\section{Introduction}

There is a high prevalence of firearm injuries in South Africa, yet data on the total number of gunshot wounds (GSWs) in South Africa is lacking. In 2005, Allard and Burch posited 127000 non-fatal GSWs occurred per annum across the country. ${ }^{1}$ Recent data from the Groote Schuur Trauma Unit's eTHR (Electronic Trauma Health Record) database shows that of the 12000 patients seen per annum, 1000 are attributable to gunshot injuries. Molinari et al. showed that $11 \%$ of gunshot-associated fractures involved the tibia. ${ }^{2}$ These tibia fractures have blast energy transferred to the bone and soft tissue, often resulting in cavitary tissue damage and a large zone of bone comminution. Additionally, in part due to the poor soft tissue coverage and close proximity of anatomical structures, tibia GSWs have an increased prevalence of neurologic deficit, higher rates of infection and delayed or non-union. ${ }^{3,4}$ These factors make these injuries difficult to treat and contribute to the controversy regarding the optimal treatment method. Because of the wide variety of GSW injury patterns, treatment plans must be personalised; therefore no single protocol can be established. ${ }^{5}$

In an attempt to minimise prosthetic infection and facilitate soft tissue management, these injuries traditionally have been treated with external fixation devices..$^{5}$ This approach protects the remaining blood supply, stabilises the bone, and allows easy access for wound care, with low infection rates. ${ }^{6}$ External fixation, however, can present issues with alignment, time to union and high rates of pin tract infection and loosening when used for extended periods.7 GSWs also have a poor correlation between external wound size and the extent of the underlying tissue damage, making predictions concerning infection and union difficult. .,, $9^{\circ}$

Intramedullary (IM) nailing is considered a viable option in treating these injuries as it provides good access to soft tissues without bulky external hardware, allows early range of motion of the knee and ankle, and causes minimal soft tissue stripping at the fracture site. ${ }^{10-12}$ Given the differing opinions on how best to treat GSWs of the tibia, this study reviewed the results of all patients treated with IM nailing of GSW tibia fractures at our tertiary care facility.

\section{Materials and methods}

A retrospective descriptive folder review was performed of all adult patients who sustained a GSW tibia fracture and were treated with intramedullary nailing between January 2009 and December 2014. All fractures with intra-articular extension or Gustilo-Anderson (GA) Grade 3B and C were excluded as these fractures are not managed with IM nailing in our unit as there is good evidence that IM nailing of these fractures yields poor results. The higher grade $3 \mathrm{~B}$ and $3 \mathrm{C}$ fractures are treated with external fixation if the limb is viable, or a staged amputation if the limb is not.
Emergency management of the fracture followed hospital protocol, which included antibiotic prophylaxis using a single dose of 2.4 million units of intramuscular penicillin and $1 \mathrm{~g}$ of intravenous Cefazolin every eight hours until wound closure. Clindamycin was used for penicillin-allergic patients. Emergency room care also included wound irrigation with normal saline or sterile water, and sterile gauze dressings under an above-knee backslab.

Operative management included thorough wound washout and debridement as needed, followed by locked, reamed intramedullary nailing under image intensification with a tibial nail (Smith \& Nephew, Trigen, Memphis, USA). Primary wound closure was performed if possible, with a negative pressure dressing applied overlying the closure, whether complete or partial, at the discretion of the operating surgeon or as interim cover. If only partial wound closure was performed the vacuum dressing was used until definitive closure. Prophylactic antibiotics were continued until 24 hours following definitive wound closure.

Post-operative mobilisation on crutches with toe-touch weight bearing together with active and passive knee and ankle range of motion exercises were started immediately, limited only by patients' pain. Partial weight bearing was encouraged for six weeks post-operatively followed by full weight bearing as able (Figures 1 and 2).

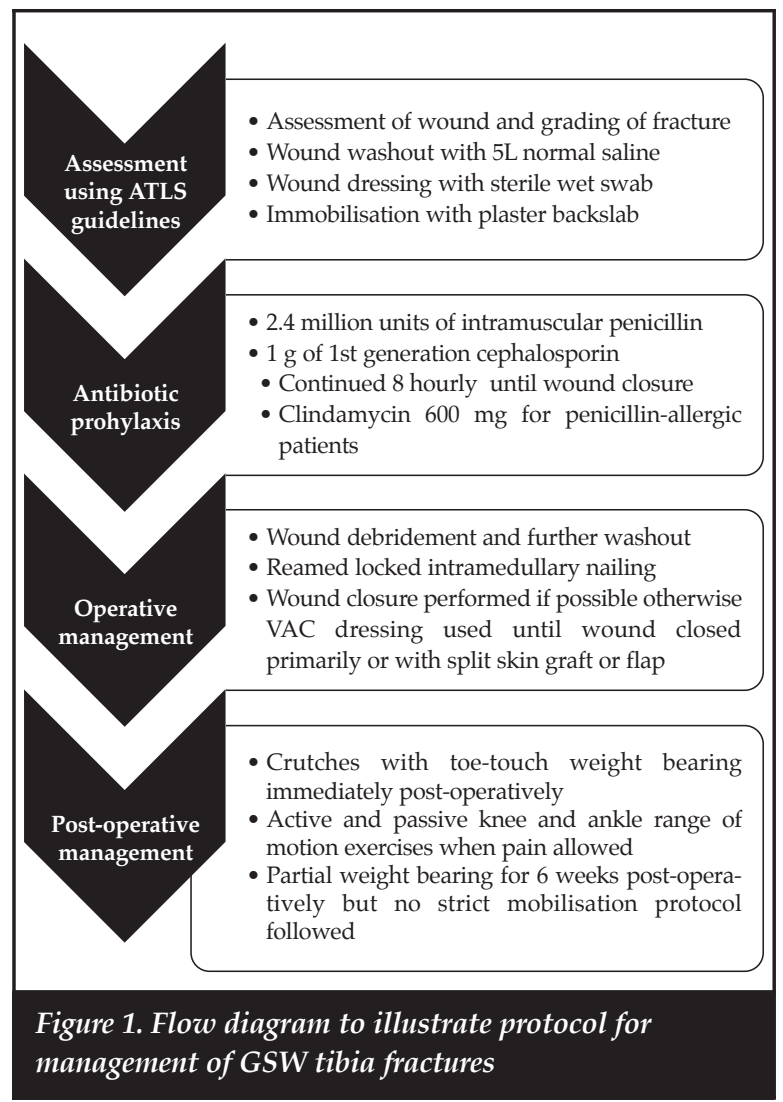




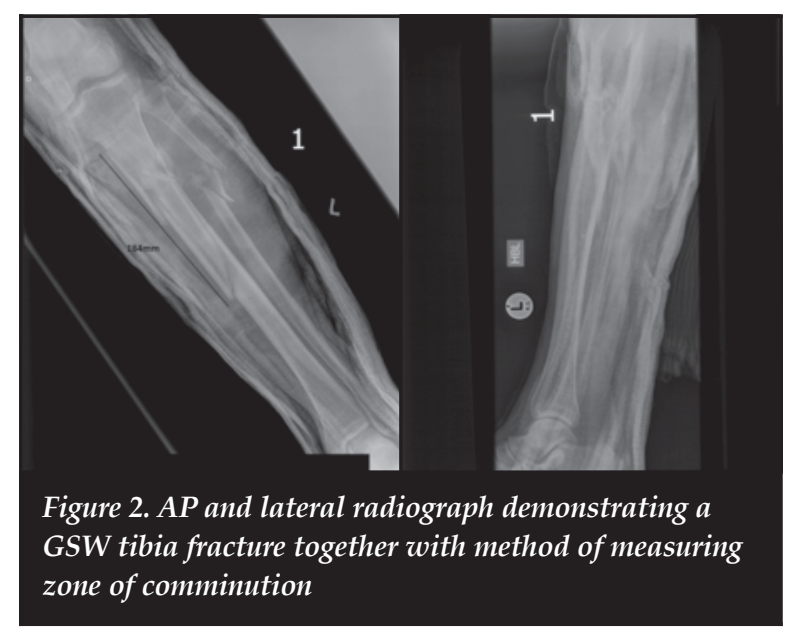

Patients were followed up until clinical and radiological union. Time to theatre, extent of fracture comminution, time to union, fibular competency, fracture grading and time to wound closure were documented, along with adverse outcomes which included deep infection or hardware failure. Parameter definitions are listed in Table I.

\section{Results}

A total of 22 patients were included. Nine were excluded from the study due only to incomplete note-taking and loss to follow-up. Of the 13 patients followed to union, 11 were male and two female. The average age of the patients was 35 years (range 22-55). The median delay to theatre from the time of the incident was 32 hours (range 5-106). All patients had definitive IM nailing at the index surgery. In eight cases the fibula was also fractured.

Five of the 13 patients had definitive closure at the index procedure, and in the remaining eight closure was achieved between three and eight days following the index procedure. Ten patients had a negative pressure wound therapy dressing applied following the index procedure. Five were overlying a definitive primary closure not requiring a second procedure; four were closed secondarily following interim negative pressure dressing cover to aid swelling subsidence and one required a small skin graft. Acceptable sagittal, coronal and rotational anatomic alignment was achieved in all patients as per the definitions in Table I. The median extent of radiographic fracture comminution was $101 \mathrm{~mm}$ (range 36-218 mm).

The average time to union was 26 weeks (range 10-70 weeks). No nerve injuries or vascular injuries requiring revascularisation were observed in this cohort. According to the Gustilo-Anderson classification, there were six grade 1 fractures, six grade 2 fractures and one grade $3 \mathrm{~A}$ fracture. No cases of hardware failure were observed. Three of the 13 cases (23\%) developed osteomyelitis. All three cases were endosteal and had their infections suppressed with oral antibiotics until union. These patients then received a second procedure to remove their nails, aggressively ream the endosteum, take multiple cultures and insert an antibiotic impregnated cement nail. This was subsequently removed after six weeks. Culturespecific antibiotics were continued for six weeks following surgery. No recurrence of infection was observed in any of these patients (Table II).

Line graphs to show trends relating fracture grade and zone of comminution to time to union are included (Figures 3 and 4).

\section{Results regarding time to union}

To compare fracture grade to time to union data the KruskalWallis test was used. A P-value of $0.010(<0.05)$ was found which shows a strong association between an increased fracture grade and a longer union time (Figure 3).

The Spearman's correlation coefficient was used to compare zone of comminution with time to union (Figure 4). A very weak Spearman's correlation coefficient value of 0.432 was found, suggesting a very mild positive correlation between a larger zone of comminution and prolonged time to union. A fractured fibular was not shown to increase the time to union with a P-value of 0.661 $(<0.05)$ when using the Mann-Whitney test.

\section{Table I: Definition of terms}

\begin{tabular}{|c|c|}
\hline Time to theatre & Time to theatre from the time of incident, calculated in hours \\
\hline $\begin{array}{l}\text { Extent of fracture } \\
\text { comminution }\end{array}$ & $\begin{array}{l}\text { The radiographic distance of fracture extent, measured along the anatomical axis from the most proximal } \\
\text { fracture line to the most distal fracture line, and recorded in millimetres on the AP radiograph }\end{array}$ \\
\hline Chronic osteomyelitis & As defined by Lew and Waldvogel, it is the presence of a biofilm-based infection, present for more than 10 days ${ }^{13}$ \\
\hline Time to closure & Time from first theatre episode to definitive closure, calculated in days \\
\hline Anatomical alignment & $\begin{array}{l}\text { Acceptable parameters for length, alignment and rotation were defined as follows: } \\
\text { - Length: within } 20 \mathrm{~mm} \text { of the unaffected side } \\
\text { - Alignment: } 5^{\circ} \text { varus or valgus. } 10^{\circ} \text { pro- or recurvatum } \\
\text { - Rotation: Neutral to } 10^{\circ} \text { of external rotation }\end{array}$ \\
\hline Time to union & $\begin{array}{l}\text { Defined as both clinical and radiographic union } \\
\text { - Clinical union: full weight bearing without assistance in the absence of pain in the affected limb } \\
\text { - Radiological union: bridging callus across three cortices }\end{array}$ \\
\hline
\end{tabular}


Table II: Summary of outcome parameters in the clinical cohort

\begin{tabular}{|c|c|c|c|c|c|c|c|}
\hline & $\begin{array}{c}\text { Time to } \\
\text { theatre (hrs) }\end{array}$ & $\begin{array}{l}\text { Extent of fracture } \\
\text { comminution }(\mathrm{mm})\end{array}$ & $\begin{array}{l}\text { Time to union } \\
\text { (weeks) }\end{array}$ & $\begin{array}{l}\text { Fibula } \\
\text { competent }\end{array}$ & $\begin{array}{l}\text { Fracture } \\
\text { grading }\end{array}$ & $\begin{array}{l}\text { Time to wound } \\
\text { closure (days)* }\end{array}$ & Osteomyelitis \\
\hline Patient 1 & 9 & 218 & 28.9 & Yes & 2 & 3 & No \\
\hline Patient 2 & 62 & 46 & 14.4 & Yes & 1 & 3 & No \\
\hline Patient 3 & 13 & 136 & 13.6 & No & 1 & 1 & Yes \\
\hline Patient 4 & 18 & 101 & 46.1 & No & 3 & 1 & No \\
\hline Patient 5 & 15 & 87 & 17.0 & No & 1 & 1 & No \\
\hline Patient 6 & 66 & 50 & 13.3 & Yes & 1 & 4 & No \\
\hline Patient 7 & 5 & 122 & 70.1 & No & 2 & 4 & Yes \\
\hline Patient 8 & 15 & 89 & 23.3 & No & 2 & 1 & No \\
\hline Patient 9 & 9 & 143 & 21.6 & Yes & 2 & 1 & No \\
\hline Patient 10 & 106 & 87 & 17.3 & No & 1 & 3 & No \\
\hline Patient 11 & 16 & 121 & 35.1 & No & 2 & 4 & Yes \\
\hline Patient 12 & 66 & 79 & 10.0 & No & 1 & 3 & No \\
\hline Patient 13 & 21 & 36 & 24.6 & Yes & 2 & 8 & No \\
\hline
\end{tabular}

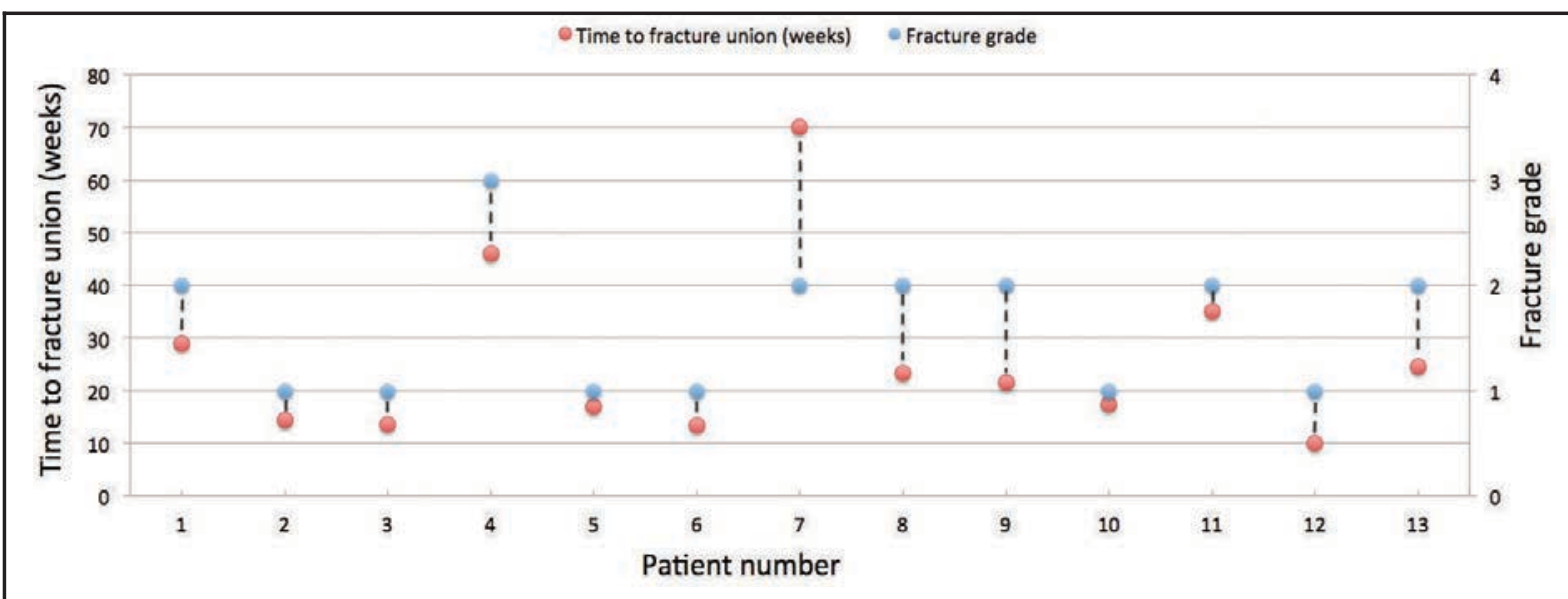

Figure 3. A graph to show the relationship of fracture grade to time to union per patient

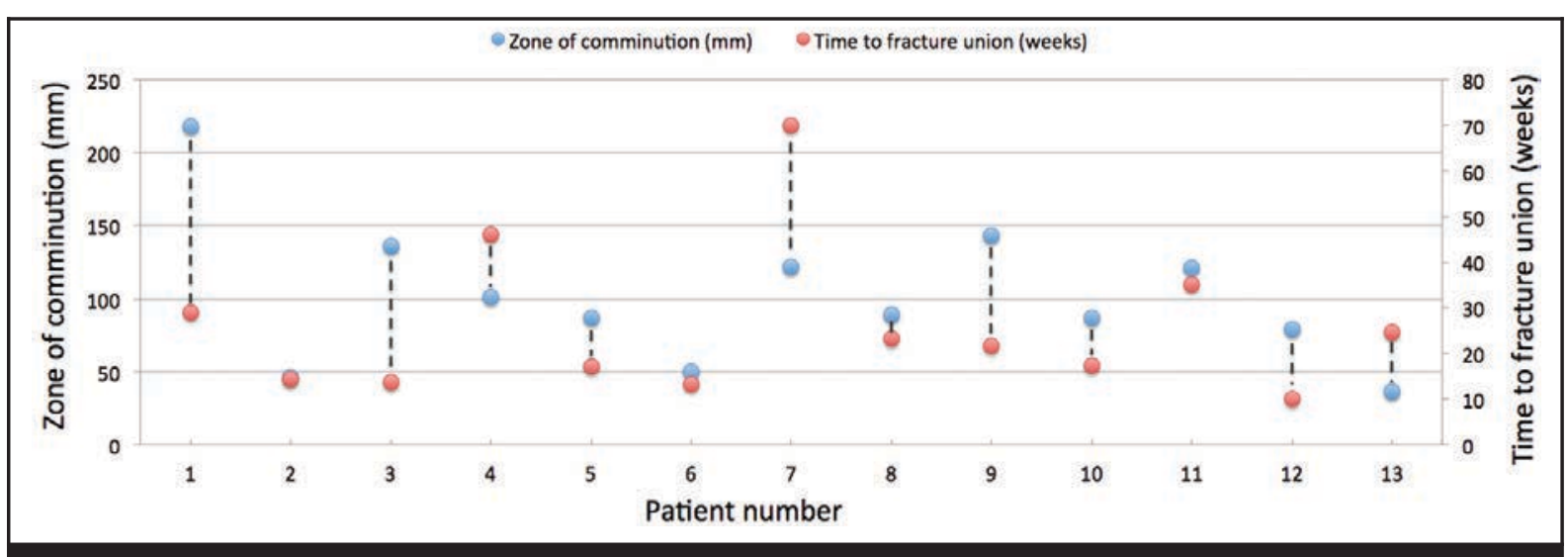

Figure 4. A graph to show the relationship of fracture zone of comminution to time to union per patient 


\section{Results regarding osteomyelitis}

Of the three cases that became infected, notably all three had radiographic zones of comminution in excess of $120 \mathrm{~mm}$. However, two other patients, also with zones of comminution in excess of $120 \mathrm{~mm}$, did not develop infection. Otherwise no trends relating time to theatre, time to union, fibular competency, fracture grade, and time to wound closure were observed.

The independent t-test was used to compare zone of fracture comminution $(\mathrm{mm})$ to probability of developing osteomyelitis. A p-value of $0.092(<0.05)$ was found showing no strong statistical significance.

For not normally distributed non-parametric data the Mann-Whitney test was used.

- Comparing effect of time to theatre (hrs) to probability of developing osteomyelitis. Outcome: P-value 0.127 $(<0.05)$.

- Comparing effect of time to union (weeks) to probability of developing osteomyelitis. Outcome: P-value $0.310(<0.05)$.

- Comparing effect of time to wound closure (Days) to probability of developing osteomyelitis. Outcome: P-value $0.478(<0.05)$.

For categorical variables, which included the fracture grade to the probability of developing osteomyelitis, the chisquared test was used. This showed a P-value of 0.672 $(<0.05)$ which shows no statistical correlation.

\section{Discussion}

Much has been made in the literature about the importance of understanding the ballistics of GSW fractures in order to guide treatment of these injuries. ${ }^{10,14-16}$ Their destructive nature results in problems with union and infection. The magnitude of tissue damaged by a bullet depends on its velocity, mass, size, shape, tumbling features and the character of tissue it strikes. There is little doubt that bullets may cause a large zone of injury with multiple bone fragments, periosteal stripping and severe soft tissue damage.

However not all GSWs of long bones, especially GSW tibia fractures,$^{17}$ are the same. The weapon used is often unknown and practically there is often no correlation between the transfer of energy and the velocity of the projectile. Therefore, the surgeon should 'treat the wound, not the weapon' ${ }^{5,14}$

This also creates controversy around the appropriate classification to use in these fractures. Gustilo and Anderson in their original article describe a 'special category' within grade 3 fractures for gunshot injuries, with no mention of them falling into grades 1 or $2 .^{18}$ Pure classification by wound size is fraught, for the underlying soft tissue and bone injury may be significant, and probably results in inaccurate classification of these injuries. This was reflected in our study, with the six classified grade 1 fractures having zones of comminution ranging from $46-136 \mathrm{~mm}$, indicating significantly higher energy transfer than normally attributed to grade 1 injuries. An alternative classification by Long et al., specifically designed for gunshot-associated femur fractures, may be more appropriate..$^{19}$ It takes into account both the physical wound characteristics and the radiographic zone of injury of both the skin and soft tissue, and degree of comminution. They do however state that 'comminution is common in low-velocity gunshot fractures of the femur and should not be used alone as the criteria to diagnose a high-velocity gunshot wound' ${ }^{19}$ One difficulty in thus applying this classification system to gunshot associated fractures of the tibia is the identification of the skin and soft tissue injury over the entire anterior and anteromedial tibial border. This region is subcutaneous and classification of the injury here will be very difficult.

In the past, tibia fractures caused by GSWs were almost exclusively treated by external fixation due to concerns about access to soft tissues and infection. IM nailing though has become the standard procedure for the treatment of closed fractures of long bones with high healing and low complication rates. ${ }^{7,12,20-22}$ With the advent of modern tibial nails, improved ballistics understanding and patient preference for an intramedullary device, more open fractures, including GSW fractures, are nailed.

A meta-analysis by Fang et al. ${ }^{7}$ compared the results of unreamed IM nailing to external fixation of open Gustilo grade 3 tibia fractures. IM nailing was shown to have distinct advantages over external fixation in many respects. These included equal if not improved access to soft tissues, no increased risk of deep infection, no external hardware, no increased delayed or non-union and a lower risk of malunion and hardware failure.

\section{Infection}

Deep infection rates for open fractures of the tibia depend largely on the degree of soft tissue injury and grade of fracture. Deep tissue infections occur only $0-2 \%$ of the time in properly treated Gustilo grade 1 and 2 open tibia fractures, but these rates jump to $21-33 \%$ for grade 3B fractures. ${ }^{4,16,20,23,24}$ Literature on the treatment of GSW tibia fractures, whether by external fixation or IM nailing is sparse, with studies mostly containing small numbers and poor follow-up. For a comparison of the different methods of treatments and their respective infection rates, see Table III.

Table III: Comparison of infection rates between treatment modalities ${ }^{16,24,25}$

Type of injury $\quad$ IM nail External fixation

\begin{tabular}{|l|l|l|}
\hline Closed (Feraro \& Zinar 1995) & $0-11 \%$ & $0-100 \%$ pin tract infection reported \\
\hline Open & $21-33 \%$ & $13 \%$ \\
\hline GSW (O'Brien et al. 1995) & $16 \%$ & $20 \%^{16}$ \\
\hline GSW (Hilton et al.) & $23 \%$ & N/A \\
\hline Exchange nailing $(<3$ weeks) & $44 \%$ & N/A \\
\hline
\end{tabular}


Deep infection for external fixation of GSW tibia fractures is relatively infrequent with most figures quoted for open tibia fractures of any mechanism, not specifically GSW tibia fractures. Only a dated article by Leffers et al. ${ }^{3}$ in 1985 mentions rates of infection for GSW tibias specifically. In their study, eight of 41 patients (19.5\%) developed a chronic discharge after surgery. Notably all their patients were either managed conservatively in a full-length plaster or with external fixation. Pin-tract infections and subsequent loosening in GSW tibia fractures are more prevalent, with rates of $36 \%$ reported by Atesalp et al. ${ }^{25}$ and $14 \%$ in the meta-analysis of Fang et al. ${ }^{7}$ Exchange nailing has also been tried but with high infection rates $(44 \%)$ if performed within the first three weeks. ${ }^{26}$ Sanders $e t$ al. ${ }^{26}$ recommended delayed exchange nailing but only in the uninfected, nutritionally balanced patient.

In studies specific to GSW of the tibia and IM nailing, variable rates of infection have been reported. Brien $e t$ al. ${ }^{16}$ described a deep infection rate of between 0 and $13 \%$ for GSW tibia fractures that underwent IM nailing. The variance in infection rates in his study was due to the grade of soft tissue injury and whether a reamed or unreamed nail was used with unreamed nails having a lower infection rate. In their meta-analysis however, Fang et al. ${ }^{7}$ disproved the difference in reaming and infections rates, and consequently, reamed nails now are used more commonly. To our knowledge, the only other study looking at infection rates of GSW tibia fractures treated with IM nailing is by Ogunlusi et al., ${ }^{12}$ who reported a $16 \%$ infection rate. When comparing, rates of deep infection are not higher in GSW tibia fractures than those of open tibia fractures caused by other mechanisms. The rate of infection is also no different when comparing open grade 3 tibia fractures treated by external fixation and IM nailing, ${ }^{7}$ but the higher energy injuries do more often develop deep infection. ${ }^{3}$

At $23 \%$, this study's deep infection rate was higher than that reported in the literature. Due to low numbers, only trends may be inferred by this study. The higher energy nature of GSW tibia fractures, however, frequently resulted in a large zone of comminution, which likely predisposed patients to the development of infection. Although widely used, the Gustilo-Anderson classification does not account for zone of comminution, which may make it a poor system to use to guide decision-making between IM nailing and external fixation in GSW tibia fractures. We suggest that the zone of comminution be weighed as significantly as the size of the soft tissue wound when determining treatment. Although further studies with larger patient numbers would be required to determine the exact size of the zone of comminution at which point external fixation would lead to fewer infections than intramedullary nailing, strong consideration should be given to definitive treatment using external fixation for fractures with zones in excess of $120 \mathrm{~mm}$.

\section{Union: Malunion, delayed and non-union}

Due to its unique anatomical characteristics, the tibia is at increased risk for malunion, delayed union, and non-union, especially with GSW tibial injuries. Ferraro et al..$^{27}$ describes a union rate for unreamed tibia IM nailing of 18 weeks (range 12-32 weeks) versus 27 weeks (range 16-64 weeks) for their external fixator group. Of their five non-unions, three occurred after external fixation, one after IM nailing and one after non-operative casting. All went on to union after revision surgery. In another study of 31 patients, Ogunlusi et al. ${ }^{12}$ showed that only one of the 31 patients, who had a IM nail for a GSW tibia fracture, did not unite within three to four months of the surgery.

Malunion rates in nailed tibial fractures, regardless of mechanism, with large zones of comminution or fractures proximal or distal to the tibial isthmic canal, have a $13 \%$ chance of greater than $5^{\circ}$ of varus or valgus malalignment. ${ }^{4}$ IM nailing has reduced rates of malunion relative to external fixation when treating open tibial fractures. ${ }^{7}$ Additional concerns over union and alignment include the presence of an intact fibula, which prevents natural dynamisation of the fracture over the nail and accurate alignment. ${ }^{3,28}$ Should delayed union occur in this scenario, Bonnevialle et al. ${ }^{28}$ advise dynamisation of the fracture over the nail with partial segment fibulectomy. In Ferarro's study, the only case of a malunion occurred in a patient with an intact fibula who was treated with an external fixator and united after a fibular corticotomy and tibial osteoclasis.

All of the patients in this study united, and those that had a delayed union showed a trend towards larger zones of comminution, fracture grade and infection (Table II, Figures 3 and 4). None of the delayed unions in this study required treatment, and none of our patients developed a malunion after their intramedullary nailing.

One of the main limitations of this and all studies of IM nailing in GSW tibias is their small number of patients. This is due in part to the narrow inclusion criteria. The patients that are eligible to be included in such a study are a subset of subset: i.e. open tibia fractures and GSW tibia fractures that have a low enough GA grading to be eligible for IM nailing. Compounding this issue is the poor follow-up that is due to economic reasons of transportation and time off work; and the social problem of gang membership prevalent among this group of patients, which causes many to avoid institutional visits as far as possible. Although we are not able to prove that the patients do not return for follow-up due to satisfactory healing and function, it is our feeling none-theless. It is possible this study experienced a drop-out rate of $40.9 \%$ because the method of fixation is load sharing and allows for early weight bearing and reduced need for close follow-up.

\section{Conclusion}

Few studies to date have specifically assessed GSW tibia fractures treated with IM nailing due to in part the limitations mentioned above. GSW tibial fractures with a GA 
grading below a $3 \mathrm{~A}$ treated with an IM nail showed equivalent results, with regard to union and infections rates, to other causes of open fractures managed in the same way. This study showed an association between grade of fracture and prolonged time to union and the zone of comminution and prolonged time to union, and this should be anticipated in GSW tibia patients treated with an IM nail. There was a trend towards increased infection rates when the radiographic zone of comminution exceeded $120 \mathrm{~mm}$. Observationally we caution the use of an intramedullary device when dealing with zones of comminution in excess of $120 \mathrm{~mm}$.

\section{Acknowledgement}

Dr Amir Ardakani, MBBS, BCs, of the Royal National Orthopaedic Hospital, Stanmore, United Kingdom, for his contribution.

\section{Compliance with Ethics Guidelines Conflict of interest statement}

Each author certifies that he or she has no commercial associations that might pose a conflict of interest in connection with the submitted article.

\section{Ethics review committee statement}

Formal approval for this study was obtained from the Human Research Ethics Committee at the University of Cape Town. Work for the article was performed at Groote Schuur Hospital.

\section{References}

1. Allard D, Burch VC. The cost of treating serious abdominal firearm-related injuries in South Africa. $S$ Afr Med J. 2005;95(8):591-94.

2. Molinari RW, Yang EC, Strauss E, Einhorn TA. Timing of internal fixation in low-velocity extremity gunshot fractures. Contemp Orthop. 1994;29(5):335-39.

3. Leffers D, Chandler RW. Tibial fractures associated with civilian gunshot injuries. The Journal of trauma. 1985;25(11):1059-64.

4. Koval KJ, Clapper MF, Brumback RJ, et al. Complications of reamed intramedullary nailing of the tibia. J Orthop Trauma. 1991;5(2):184-89.

5. Crainz E, Gambera D, Maniscalco P, Bertone C, Rivera F, Maggiore D. Low-velocity gunshot fractures of the tibia. Journal of orthopaedic science : official journal of the Japanese Orthopaedic Association. 2002;7(3):386-91.

6. Mandracchia VJ, Buddecke DE, Jr., Statler TK, Nelson SC. Gunshot wounds to the lower extremity. A comprehensive review. Clin Podiatr Med Surg. 1999;16(4):597-615.

7. Fang X, Jiang L, Wang $Y$, Zhao L. Treatment of Gustilo grade III tibial fractures with unreamed intramedullary nailing versus external fixator: a meta-analysis. Med Sci Monit. 2012;18(4):RA49-56

8. Hull JB. Management of gunshot fractures of the extremities. J Trauma. 1996;40(3 Suppl):S193-97.

9. Hollmann MW, Horowitz M. Femoral fractures secondary to low velocity missiles: treatment with delayed intramedullary fixation. J Orthop Trauma. 1990;4(1):64-69.
10. Dicpinigaitis PA, Fay R, Egol KA, Wolinsky P, Tejwani N, Koval KJ. Gunshot wounds to the lower extremities. American Journal of Orthopedics (Belle Mead, NJ). 2002;31(5):282-93.

11. Sanders R, Swiontkowski MF, Nunley JA, 2nd, Spiegel PG. The management of fractures with soft-tissue disruptions. Instr Course Lect. 1994;43:559-70.

12. Ogunlusi JD, Olasinde A, Ikem IC, Davids T. Gunshot fractures of tibia and femur - excellent results with reamed bone marrow graft and interlocking nailing. Vol 882011:337-43.

13. Lew DP, Waldvogel FA. Osteomyelitis. $N$ Engl J Med. 1997;336(14):999-1007.

14. Dougherty PJ, Sherman D, Dau N, Bir C. Ballistic fractures: indirect fracture to bone. Journal of Trauma. 2011;71(5):1381-84.

15. Griffiths DC, J. (iii) Military limb injuries/ballistic fractures. Current Orthopaedics. 2006;20(5):346-53.

16. Brien EW, Long WT, Serocki JH. Management of gunshot wounds to the tibia. Orthopedic Clinics of North America. 1995;26(1):165-80.

17. Afonso S, O'Brien GS, Jaramillo CV, McKenna DH, Rowe GG. Left ventricular heat production after lowering left ventricular work. Am J Physiol. 1966;210(3):553-56.

18. Gustilo RB, Anderson JT. Prevention of infection in the treatment of one thousand and twenty-five open fractures of long bones: retrospective and prospective analyses. J Bone Joint Surg Am. 1976;58(4):453-58.

19. Long WT, Chang W, Brien EW. Grading system for gunshot injuries to the femoral diaphysis in civilians. Clin Orthop Relat Res. 2003(408):92-100.

20. Malik MH, Harwood P, Diggle P, Khan SA. Factors affecting rates of infection and nonunion in intramedullary nailing. J Bone Joint Surg Br. 2004;86(4):556-60.

21. Seyhan $M$, Unay $K$, Sener N. Intramedullary nailing versus percutaneous locked plating of distal extra-articular tibial fractures: a retrospective study. Eur J Orthop Surg Traumatol. 2013;23(5):595-601.

22. Robinson CM, McLauchlan G, Christie J, McQueen MM, CourtBrown CM. Tibial fractures with bone loss treated by primary reamed intramedullary nailing. I Bone Joint Surg $\mathrm{Br}$. 1995;77(6):906-13.

23. Wiss DA, Brien WW, Becker V, Jr. Interlocking nailing for the treatment of femoral fractures due to gunshot wounds. J Bone Joint Surg Am. 1991;73(4):598-606.

24. Wiss DA, Stetson WB. Unstable fractures of the tibia treated with a reamed intramedullary interlocking nail. Clinical orthopaedics and related research. 1995;(315)(315):56-63.

25. Atesalp AS, Komurcu M, Demiralp B, Bek D, Oguz E, Yanmis I. Treatment of close-range, low-velocity gunshot fractures of tibia and femur diaphysis with consecutive compression-distraction technique: a report of 11 cases. Journal of Surgical Orthopaedic Advances. 2004;13(2):112-18.

26. Sanders R, Swiontkowski M, Nunley J, Spiegel P. The management of fractures with soft-tissue disruptions. J Bone Joint Surg Am. 1993;75(5):778-89.

27. Ferraro SPJ, Zinar DM. Management of gunshot fractures of the tibia. The Orthopedic Clinics of North America. 1995;26(1):181-89.

28. Bonnevialle P, Bellumore Y, Foucras L, Hezard L, Mansat M. [Tibial fracture with intact fibula treated by reamed nailing]. Rev Chir Orthop Reparatrice Appar Mot. 2000;86(1):29-37.

This article is also available online on the SAOA website (www.saoa.org.za) and the SciELO website (www.scielo.org.za). Follow the directions on the Contents page of this journal to access it. 